\title{
Erratum: Relativistic mean-field interaction with density-dependent meson-nucleon vertices based on microscopical calculations [Phys. Rev. C 84, 054309 (2011)]
}

\author{
X. Roca-Maza, X. Viñas, M. Centelles, P. Ring, and P. Schuck \\ (Received 8 June 2016; published 27 June 2016)
}

DOI: 10.1103/PhysRevC.93.069905

We have recently realized that the significant digits reported in Table I of the original paper for the parameters of the DD-ME $\delta$ interaction are not sufficient to accurately reproduce the results published in the same paper. The reason can be easily understood by considering that, for example, in the prediction of masses, there exists a strong cancellation between the contributions of the different meson fields that can be, separately, very large [1]. Specifically, we have checked that one would need to use the value of the parameters with seven significant digits. In Table I below, we give all the parameters of the DD-ME $\delta$ functional with seven significant digits.

TABLE I. The parameter set DD-ME $\delta$. The difference of this table with Table I in the original paper is that we report here seven significant digits.

\begin{tabular}{lcccccr}
\hline \hline$i$ & $m_{i}(\mathrm{MeV})$ & $g_{i}\left(\rho_{\text {sat }}\right)$ & $a_{i}$ & $b_{i}$ & $c_{i}$ & $d_{i}$ \\
\hline$\sigma$ & 566.1577 & 10.33254 & 1.392730 & 0.1901198 & 0.3678654 & 0.9519078 \\
$\omega$ & 783.0000 & 12.29041 & 1.408892 & 0.1697977 & 0.3429006 & 0.9859508 \\
$\delta$ & 983.0000 & 7.151971 & 1.517787 & 0.3262490 & 0.6040782 & 0.4257178 \\
$\rho$ & 763.0000 & 6.312758 & 1.887685 & 0.06514596 & 0.3468963 & 0.98595078 \\
\hline \hline
\end{tabular}

[1] P. Ring, Prog. Part. Nucl. Phys. 37, 193 (1996). 